\title{
Possible constraints on SUSY-model Parameters from Direct Dark Matter Search 1 .
}

\author{
V.A. Bednyakov, H.V. Klapdor-Kleingrothaus*, S.G. Kovalenko \\ Joint Institute for Nuclear Research, Dubna, Russia \\ *Max-Plank-Institut für Kernphysik, D-6900, Heidelberg, Germany
}

\begin{abstract}
We consider the SUSY-model neutralino as a dominant Dark Matter particle in the galactic halo and investigate some general issues of direct DM searches via elastic neutralino-nucleus scattering. On the basis of conventional assumptions about the nuclear and nucleon structure, without referring to a specific SUSY-model, we prove that it is impossible in principle to extract more than three constraints on fundamental SUSY-model parameters from the direct Dark matter searches. Three types of Dark matter detectors probing different groups of parameters are recognized.
\end{abstract}

${ }^{1}$ This work was supported in part by the Russian Foundation for Fundamental Research (93-02-3744). 
Many physicists at present believe that more than $90 \%$ of the total universe mass should be in the form of non-luminous non-baryonic dark matter (DM). Most of our galaxy mass should be in a dark halo as well. According to the commonly accepted halo model the DM particles have a Maxwellian velocity distribution in the galactic frame, a mass density in the Solar system of about $\rho \approx 0.3 \mathrm{GeV} \cdot \mathrm{cm}^{-3}$ and come to the Earth with mean velocities $v \approx 320 \mathrm{~km} / \mathrm{sec}$, producing a substantial flux $\Phi=\rho \cdot v / M\left(\Phi>10^{7} \mathrm{~cm}^{-2} \mathrm{sec}^{-1}\right.$ for a particle mass $M \sim 1 \mathrm{GeV}$ ). Thus the idea of detecting DM particles directly, for instance through the elastic scattering off nuclei in detector target material looks realistic.

Dark Matter in the universe consists of two main components, which play different cosmological roles. These are "hot" (HDM) and "cold" (CDM) DM components which decoupled from the thermal equilibrium in the early universe at relativistic and non-relativistic temperatures, respectively. The analysis of the COBE satellite data [1] on anisotropy in the cosmic background radiation and the theory of the formation of large scale structure in the universe lead to the following DM composition $\mathrm{DM}=0.7(\mathrm{CDM})+0.3(\mathrm{HDM})$ [2], [3]. It is commonly believed that the CDM is represented by weakly interacting massive particles (WIMP) and the axion, while the HDM consists of light neutrinos. We do not discuss the axion as a DM candidate, concentrating on the pure WIMP variant of the CDM.

The neutralino $(\chi)$ is a SUSY-model version of WIMP. Presently it is the most favorable CDM candidate. $\chi$ is a Majorana $\left(\chi^{c}=\chi\right)$ particle with spin $\frac{1}{2}$ and usually assumed to be the lightest SUSY-particle (LSP).

The problem of direct detection of the DM neutralino $\chi$ via elastic scattering off nuclei has been considered by many authors and remains a field of great experimental and theoretical activity [4]- [13].

One can meet in the literature the statement that the neutralino prefers to scatter off spin-non-zero $(\mathrm{J} \neq 0)$ nuclei due to its Majorana nature. However, we have shown [13] that for sufficiently heavy nuclei nuclear spin is a non-dominant factor in the neutralino-nucleus scattering. Thus, one has equal chances to detect the DM signal using either spin-zero or spin-non-zero target nuclei with atomic weight $A>50$.

However, $\mathrm{J} \neq 0$ and $\mathrm{J}=0$ - nuclei provide different information about SUSYmodel parameters. Therefore experiments with $\mathrm{J} \neq 0$ and with $\mathrm{J}=0$ - nuclei are both important from the point of view of SUSY-phenomenology.

In the present paper we envisage the question of how many independent constraints on fundamental SUSY-model parameters can be extracted from direct DM neutralino searches. On the basis of conventional assumptions we shall prove that there are just three independent constraints for special groups of parameters. 
In this respect we can distinguish three types of DM detectors with different sensitivity to the groups of fundamental parameters. These are detectors with non-zero-spin nuclei of neutron-like $\left({ }^{3} \mathrm{He},{ }^{29} \mathrm{Si},{ }^{73} \mathrm{Ge}, \ldots\right)$ and proton-like $\left({ }^{19} \mathrm{~F}\right.$, $\left.{ }^{35} \mathrm{Cl},{ }^{205} \mathrm{Tl}, \ldots\right)$ shell-model structure, and spin-zero nuclei $\left({ }^{76} \mathrm{Ge}, \ldots\right)$.

The DM event is elastic neutralino-nucleus scattering causing a nuclear recoil detected by detector. The event rate per unit mass of target material depends on the density and velocity of DM in the solar vicinity and the cross section $\sigma_{e l}(\chi A)$ of the neutralino-nucleus $(\chi A)$ elastic scattering. One can calculate $\sigma_{e l}(\chi A)$ starting from the neutralino-quark effective Lagrangian. It can be written in the general form as

$$
L_{e f f}=\sum_{q} \mathcal{A}_{q} \cdot \bar{\chi} \gamma_{\mu} \gamma_{5} \chi \cdot \bar{q} \gamma^{\mu} \gamma_{5} q+\frac{m_{q}}{M_{W}} \cdot \mathcal{S}_{q} \cdot \bar{\chi} \chi \cdot \bar{q} q
$$

where terms with the vector and pseudoscalar quark currents are omitted being negligible in the case of non-relativistic DM neutralinos with typical velocities $v_{\chi} \approx 10^{-3} c$.

The coefficients $\mathcal{A}_{q}, \mathcal{S}_{q}$ are effective phenomenological parameters completely parametrizing low-energy neutralino-quark interactions. They can be calculated in a specific SUSY-model. Furtheron we will consider the most general properties of the $\chi$ - $A$ scattering starting from the effective Lagrangian (1) without referring to specific SUSY-models. To calculate the elastic neutralino-nucleus cross section $\sigma_{e l}(\chi A)$ we follow the standard procedure of sequentially averaging the $\chi-q$ interactions over the nucleon and then the nuclear structure.

The first and the second terms in $L_{\text {eff }}$ (1) averaged over the nucleon states give spin-dependent and spin-independent matrix elements $M_{s d}$ and $M_{s i}$, respectively. For the spin-dependent matrix element we have [4], [5]:

$$
M_{s d}^{p(n)}=4 \vec{S}_{\chi} \vec{S}_{p(n)} \cdot \mathcal{W}_{p(n)}
$$

where $\vec{S}_{\chi}$ and $\vec{S}_{p(n)}$ are the neutralino and proton (neutron) spin-operators. The factor $\mathcal{W}_{p(n)}$ depending on the parameters of the effective Lagrangian (看) has the form

$$
\mathcal{W}_{p(n)}=\sum_{q \in p(n)} \mathcal{A}_{q} \Delta q
$$

$\Delta q$ are the fractions of the nucleon spin carried by the quark $q$. The definition is

$$
<p(n)\left|\bar{q} \gamma^{\mu} \gamma_{5} q\right| p(n)>=2 S_{p(n)}^{\mu} \Delta q
$$

where $S_{p(n)}^{\mu}=\left(0, \vec{S}_{p(n)}\right)$ is the 4 -spin of the nucleon. The parameters $\Delta q$ for the proton can be extracted from the EMC [14] and hyperon data [15]:

$$
\Delta u=0.77 \pm 0.08, \quad \Delta d=-0.49 \pm 0.08, \quad \Delta s=-0.15 \pm 0.08
$$


The relevant values for the neutron can be found from (5) by isospin symmetry substitution $u \rightarrow d, d \rightarrow u$.

The spin-independent matrix element $M_{s i}$ has the form [7], [8]:

$$
M_{s i}=\frac{M_{p(n)}}{M_{W}} \cdot \bar{\chi} \chi \cdot \bar{\Psi} \Psi \cdot \mathcal{V} .
$$

Again as in the spin-dependent matrix element (2) we separate the factor

$$
\mathcal{V}=\sum_{q} f_{q} \cdot \mathcal{S}_{q}
$$

where the parameters $f_{q}$ are defined as follows:

$$
<p(n)\left|m_{q} \cdot \bar{q} q\right| p(n)>=f_{q} \cdot M_{p(n)} \bar{\Psi} \Psi .
$$

The values extracted from the data are [16], [17]:

$$
\begin{gathered}
f_{u}=0.022, \quad f_{d}=0.034, \quad f_{s}=0.38, \\
f_{c}=f_{b}=f_{t}=\frac{2}{27}\left(1-f_{u}-f_{d}-f_{s}\right) .
\end{gathered}
$$

The next step is the calculation of nuclear matrix elements of scalar and axialvector nucleon operators $\bar{\Psi} \cdot \Psi$ and $\bar{\Psi} \gamma_{\mu} \gamma_{5} \Psi$, respectively for the nuclear state $\mid A>$. The axial-vector operator corresponding in a non-relativistic limit to the nucleon spin-operator appears in eqn. (2). We can parametrize these nuclear matrix elements in the most general form as follows

$$
\begin{aligned}
<A\left|M_{p(n)} \bar{\Psi} \Psi\right| A> & =M_{A} F_{s i}\left(q^{2}\right) \bar{A} A, \\
<A\left|\vec{S}_{p(n)}\right| A> & =\lambda_{p(n)} F_{s d}^{p(n)}\left(q^{2}\right)<A|\vec{J}| A>.
\end{aligned}
$$

Here $\vec{J}$ is the nuclear spin. The form factors $F_{s d, s i}\left(q^{2}\right)$ take into account effects of finite momentum transfer $q$ and obey the normalization conditions:

$$
F_{s d, s i}(0)=1 .
$$

For nuclei with atomic weight $A>50$ nuclear structure is essential since $q_{\max } \geq$ $1 / R_{A}$ ( $R_{A}$ is the nuclear radius) [18]. In this case the form factors strongly deviate from the normalization value.

\footnotetext{
${ }^{1}$ When this paper had been completed we received a paper ref. [1] with more refined treatment of the spin-independent matrix element.
} 
The phenomenological parameter $\lambda_{p(n)}$ measures the contribution of the proton (neutron) group to the nuclear spin (See 18 for these nomenclature.). According to the conventional shell model picture, the odd-group contribution dominates for even-odd nuclei. For odd-proton group nuclei $\left({ }^{7} \mathrm{Li},{ }^{19} \mathrm{~F},{ }^{203} \mathrm{Tl}, \ldots\right)$ $\lambda_{p}>>\lambda_{n}$ and for odd-neutron group nuclei $\left({ }^{3} \mathrm{He},{ }^{29} \mathrm{Si},{ }^{73} \mathrm{Ge}, \ldots\right) \lambda_{n}>>\lambda_{p}$.

In the case of odd-odd nuclei $\left({ }^{10} \mathrm{~B},{ }^{14} \mathrm{~N}, \ldots\right)$ proton and neutron groups give comparable contributions to a nuclear spin and $\lambda_{n} \sim \lambda_{p}$.

The $\lambda$-parameters should be calculated in some nuclear model. In the semiempirical odd-group shell model [18] they can be related to the nuclear magnetic moment, $\mu$, as follows

$$
\lambda J=\frac{\mu-g^{l} J}{g^{s}-g^{l}},
$$

where $g^{l}=1(0)$ and $g^{s}=5.586(-3.826)$ are orbital and spin proton (neutron) $g$-factors.

Then one can extract $\lambda$-values from the experimental data on nuclear magnetic moments:.

For our purpose the numerical values of these parameters are irrelevant but for completeness we give them in the Appendix for nuclei of interest in the DM search.

Making standard transformations of the above-written matrix elements and averaging over the Maxwellian distribution of neutralino velocities, we finally arrive at the formula for the event rate of the elastic neutralino-nucleus scattering in the detector per day per unit mass of the target material:

$$
R=\left[a_{p} \cdot \mathcal{W}_{p}^{2}+a_{n} \cdot \mathcal{W}_{n}^{2}+a_{0} \cdot \mathcal{V}^{2}\right] \frac{\text { events }}{\mathrm{kg} \cdot \text { day }}
$$

The parameters $a_{i}$ depend on properties of the target nucleus as well as on the mass density and the average velocity of DM particles in the solar vicinity. In the Appendix we give the values of these parameters for different nuclei within specific model assumptions. The above-defined quantities $\mathcal{W}$ and $\mathcal{V}$ contain all dependence on the parameters of the effective Lagrangian (11) and do not depend on nuclear properties. This factorization is a central point of the present paper.

It follows from eqn. (12) that measuring the event rate $R$ we can study just three special combinations of fundamental parameters $\mathcal{W}_{p, n}$ and $\mathcal{V}$ defined in eqn. (3), (17). This is the only information about fundamental parameters accessible in DM search experiments. $\mathrm{R}$ is a linear combination of the quantities

\footnotetext{
${ }^{2}$ Another approach based on the theory of finite Fermi systems is described in [19].
} 
$\mathcal{W}_{p, n}^{2}$ and $\mathcal{V}^{2}$. To extract experimental limitations for each of them one should search for DM with different target nuclei. We can distinguish three types of DM detectors by their sensitivity to $\mathcal{W}_{p, n}$ and $\mathcal{V}$. These are detectors built of:

1. spin-non-zero target nuclei with an 'odd-proton group' probing a linear combination

$$
a_{p} \cdot \mathcal{W}_{p}^{2}+a_{0} \cdot \mathcal{V}^{2}<R_{e x p}^{(1)}
$$

and giving an experimental limit $R_{e x p}^{(1)}$;

2. spin-non-zero target-nuclei with an 'odd-neutron group' probing another linear combination

$$
a_{n} \cdot \mathcal{W}_{n}^{2}+a_{0} \cdot \mathcal{V}^{2}<R_{e x p}^{(2)}
$$

and giving an experimental limit $R_{e x p}^{(2)}$;

3. spin-zero target nuclei sensitive only to the scalar part of the neutralinonucleus interaction

$$
\mathcal{V}^{2}<R_{e x p}^{(3)}
$$

giving an experimental limit $R_{e x p}^{(3)}$.

In conclusion we would like to stress the following. To extract all information about SUSY-model parameters possible from direct DM search one should have three established types of DM detectors. No other information can be obtained from the direct DM search experiments. Different detectors can only improve the data on the three above-defined groups of SUSY-model parameters.

A detailed discussion on the extractable information about SUSY model parameters will be given elsewhere.

\section{Appendix}

Below we give complete formulae for calculation of the nuclear structure parameters entering in the definition of the event rate $R$ in eqn. (12) within commonly accepted model assumptions.

Assuming a gaussian parametrization [10] for the form factors $F_{s d, s i}\left(q^{2}\right)$ in eqns. (9) and averaging over the Maxwellian distribution of DM particle velocities we get

$$
\begin{aligned}
a_{p(n)} & =5.8 \cdot 10^{10} \cdot \lambda_{p(n)}^{2} J(J+1) \cdot \zeta\left(r_{\text {spin }}\right) \cdot \mathcal{D}, \\
a_{0} & =1.44 \cdot 10^{10} \cdot\left(\frac{M_{A}}{M_{W}}\right)^{2} \zeta\left(r_{\text {charge }}\right) \cdot \mathcal{D},
\end{aligned}
$$


where the DM factor is

$$
\mathcal{D}=\left[\frac{4 M_{\chi} M_{A}}{\left(M_{\chi}+M_{A}\right)^{2}}\right]\left[\frac{\rho}{.3 G e V \cdot \mathrm{cm}^{-3}}\right]\left[\frac{<\left|\overrightarrow{v_{E}}\right|>}{320 \mathrm{~km} / \mathrm{s}}\right] .
$$

Here $M_{\chi}, M_{A}$ are the DM neutralino and target nucleus masses. The coherence loss factor 20] is defined by

$$
\zeta(r)=\frac{0.573}{b}\left(1-\frac{\exp \left(-\frac{b}{1+b}\right)}{\sqrt{1+b}} \frac{\operatorname{erf}\left(\sqrt{\frac{1}{1+b}}\right)}{\operatorname{erf}(1)}\right)
$$

where

$$
b=\frac{8}{9} \sigma^{2} r^{2} \frac{M_{\chi}^{2} M_{A}^{2}}{\left(M_{\chi}+M_{A}\right)^{2}} .
$$

Here $\sigma^{2}$ is the dispersion of the Maxwellian neutralino velocity distribution $\sigma=$ $0.9 \cdot 10^{-3} \cdot r_{\text {spin }}$ and $r_{\text {charge }}$ are the rms spin and charge radius of the nucleus $A$. To estimate $r_{\text {charge }}$ one can use the parametrization [21]:

$$
r_{\text {charge }}=\left(0.3+0.89 M_{A}^{1 / 3}\right) \mathrm{fm} .
$$

The values of the ratio $r_{\text {spin }} / r_{\text {charge }}$ can be estimated in the harmonic oscillator shell model [9].

Numerical values of the above defined parameters for nuclei of interest in direct DM searches are given in the Table.

\section{References}

[1] G.F.Smoot et al, Astrophys.J. 396 (1992) L1.

[2] A.N.Taylor, M.Rowan-Robinson, Nature 359 (1992) 336.

[3] M.Davis, F.J.Summers and D.Schlegel, Nature 359 (1992) 393.

[4] M.W.Googman, E.Witten, Phys.Rev. D31 (1985) 3059.

[5] J.Ellis, R.Flores, Nucl.Phys. B307 (1988) 883.

[6] K.Griest, Phys.Rev. D38 (1988) 2357; D39 (1989) 3802.

[7] G.B.Gelmini, P.Gondolo and E.Roulet, Nucl.Phys. B351 (1991) 623.

[8] R.Flores, K.A.Olive and D.Thomas, Phys.Lett. B263 (1991) 425. 
[9] J.Ellis, R.Flores, Phys.Lett. B300 (1993) 175; CERN-TH-6588/92.

[10] J.Ellis, R.Flores, Phys.Lett., B263 (1991) 259.

[11] M. Drees, M.M. Nojiri, Univ. Wisconsin - Madison, 1993, prepr. MAD/PH/768.

[12] A.Bottino, V.de Alfaro, N.Fornengo, G.Mignola and S. Scopel, Astropart.Phys.J. 1 (1992) 61.

[13] V.A. Bednyakov, H.V. Klapdor-Kleingrothaus, S.G. Kovalenko (to be pubblished).

[14] J.Ashman et al., EMC collaboration, Nucl.Phys. B328 (1989) 1.

[15] A.Manohar, R.Jaffe, Nucl.Phys. B337 (1990) 509.

[16] T.P.Cheng, Phys.Rev. D38 (1988) 2869; H.-Y.Cheng, Phys.Lett. B219 (1989) 347.

[17] J.Gasser, H.Leutwyler and M.E. Sainio, Phys.Lett. B253 (1991) 252.

[18] J.Engel, P.Vogel, Phys.Rev. D40 (1989) 3132.

[19] M.A.Nikolaev, H.V.Klapdor-Kleigrothaus, Z.Phys. A345 (1993) 183; 373.

[20] K.Freese, J.Frieman and A.Gould, Phys.Rev. D37 (1988) 3388; A.Gould, Astrophys.J. 321 (1987) 571.

[21] G. Eder, "Nuclear Forces", MIT Press, Cambridge, 1968, p.56. 
Table: Parameters for event rate calculations.

\begin{tabular}{|r|r|l|l|r||r|r|l|l|l|}
\hline Isotope & $\mathrm{J}$ & $\lambda_{p}^{2}$ & $\lambda_{n}^{2}$ & $\frac{r_{\text {spin }}}{r_{\text {charge }}}$ & ${ }^{\text {Isotope }}$ & $\mathrm{J}$ & $\lambda_{p}^{2}$ & $\lambda_{n}^{2}$ & $\frac{r_{\text {spin }}}{r_{\text {charge }}}$ \\
\hline \hline${ }^{1} \mathrm{H}$ & $1 / 2$ & 1.0 & 0 & 1.00 & ${ }^{99} \mathrm{Ru}$ & $5 / 2$ & 0 & 0.0045 & 1.19 \\
\hline${ }^{3} \mathrm{He}$ & $1 / 2$ & 0 & 1.2373 & 1.00 & ${ }^{101} \mathrm{Ru}$ & $5 / 2$ & 0 & 0.0056 & 1.19 \\
\hline${ }^{7} \mathrm{Li}$ & $3 / 2$ & 0.1096 & 0 & 1.17 & ${ }^{107} \mathrm{Ag}$ & $1 / 2$ & 0.0720 & 0 & 1.06 \\
\hline${ }^{9} \mathrm{Be}$ & $3 / 2$ & 0 & 0.0768 & 1.12 & ${ }^{109} \mathrm{Ag}$ & $1 / 2$ & 0.0760 & 0 & 1.06 \\
\hline${ }^{11} \mathrm{~B}$ & $3 / 2$ & 0.0299 & 0 & 1.09 & ${ }^{111} \mathrm{Cd}$ & $1 / 2$ & 0 & 0.0960 & 1.17 \\
\hline${ }^{15} \mathrm{~N}$ & $1 / 2$ & 0.1160 & 0 & 1.06 & ${ }^{113} \mathrm{Cd}$ & $1 / 2$ & 0 & 0.1053 & 1.17 \\
\hline${ }^{17} \mathrm{O}$ & $5 / 2$ & 0 & 0.0391 & 1.25 & ${ }^{115} \mathrm{Sn}$ & $1 / 2$ & 0 & 0.2307 & 1.16 \\
\hline${ }^{19} \mathrm{~F}$ & $1 / 2$ & 0.8627 & 0 & 1.21 & ${ }^{117} \mathrm{Sn}$ & $1 / 2$ & 0 & 0.2733 & 1.16 \\
\hline${ }^{23} \mathrm{Na}$ & $3 / 2$ & 0.0109 & 0 & 1.16 & ${ }^{121} \mathrm{Sb}$ & $5 / 2$ & 0.0057 & 0 & 1.15 \\
\hline${ }^{27} \mathrm{Al}$ & $5 / 2$ & 0.0099 & 0 & 1.13 & ${ }^{123} \mathrm{Sb}$ & $7 / 2$ & 0.0035 & 0 & 1.15 \\
\hline${ }^{29} \mathrm{Si}$ & $1 / 2$ & 0 & 0.0840 & 1.12 & ${ }^{127} \mathrm{I}$ & $5 / 2$ & 0.0026 & 0 & 1.15 \\
\hline${ }^{31} \mathrm{P}$ & $1 / 2$ & 0.0760 & 0 & 1.11 & ${ }^{129} \mathrm{Xe}$ & $1 / 2$ & 0 & 0.1653 & 1.14 \\
\hline${ }^{35} \mathrm{Cl}$ & $3 / 2$ & 0.0096 & 0 & 1.10 & ${ }^{131} \mathrm{Xe}$ & $3 / 2$ & 0 & 0.0147 & 1.14 \\
\hline${ }^{47} \mathrm{Ti}$ & $5 / 2$ & 0 & 0.0067 & 1.20 & ${ }^{133} \mathrm{Cs}$ & $7 / 2$ & 0.0033 & 0 & 1.14 \\
\hline${ }^{49} \mathrm{Ti}$ & $7 / 2$ & 0 & 0.0068 & 1.20 & ${ }^{139} \mathrm{La}$ & $7 / 2$ & 0.0020 & 0 & 1.13 \\
\hline${ }^{51} \mathrm{~V}$ & $7 / 2$ & 0.0106 & 0 & 1.19 & ${ }^{155} \mathrm{Gd}$ & $3 / 2$ & 0 & 0.0021 & 1.22 \\
\hline${ }^{55} \mathrm{Mn}$ & $5 / 2$ & 0.0069 & 0 & 1.17 & ${ }^{157} \mathrm{Gd}$ & $3 / 2$ & 0 & 0.0035 & 1.22 \\
\hline${ }^{59} \mathrm{Co}$ & $7 / 2$ & 0.0049 & 0 & 1.15 & ${ }^{183} \mathrm{~W}$ & $1 / 2$ & 0 & 0.0040 & 1.19 \\
\hline${ }^{67} \mathrm{Zn}$ & $5 / 2$ & 0 & 0.0083 & 1.13 & ${ }^{191} \mathrm{Ir}$ & $3 / 2$ & 0.0387 & 0 & 1.08 \\
\hline${ }^{69} \mathrm{Ga}$ & $3 / 2$ & 0.0056 & 0 & 1.13 & ${ }^{193} \mathrm{Ir}$ & $3 / 2$ & 0.0379 & 0 & 1.08 \\
\hline${ }^{71} \mathrm{Ga}$ & $3 / 2$ & 0.0237 & 0 & 1.13 & ${ }^{199} \mathrm{Hg}$ & $1 / 2$ & 0 & 0.0693 & 1.17 \\
\hline${ }^{73} \mathrm{Ge}$ & $9 / 2$ & 0 & 0.0026 & 1.25 & ${ }^{201} \mathrm{Hg}$ & $3 / 2$ & 0 & 0.0096 & 1.17 \\
\hline${ }^{79} \mathrm{Br}$ & $3 / 2$ & 0.0077 & 0 & 1.11 & ${ }^{203} \mathrm{Tl}$ & $1 / 2$ & 0.2400 & 0 & 1.07 \\
\hline${ }^{81} \mathrm{Br}$ & $3 / 2$ & 0.0125 & 0 & 1.11 & ${ }^{205} \mathrm{Tl}$ & $1 / 2$ & 0.2467 & 0 & 1.07 \\
\hline${ }^{91} \mathrm{Zr}$ & $5 / 2$ & 0 & 0.0186 & 1.21 & ${ }^{207} \mathrm{~Pb}$ & $1 / 2$ & 0 & 0.0960 & 1.17 \\
\hline${ }^{93} \mathrm{Nb}$ & $9 / 2$ & 0.0065 & 0 & 1.20 & ${ }^{209} \mathrm{Bi}$ & $9 / 2$ & 0.0002 & 0 & 1.16 \\
\hline
\end{tabular}

